\title{
Reachability and Controllability of Switched Linear Discrete-Time Systems
}

\author{
S. S. Ge, ${ }^{*}$ Z. Sun and T. H. Lee \\ Department of Electrical Engineering \\ National University of Singapore \\ Singapore 117576
}

\begin{abstract}
This paper investigates the reachability and controllability issues for switched linear discrete-time systems. Geometric characterization of controllability is presented. For reversible systems, the controllable sets and the reachable sets are identified in Wonham's geometric approach, and verifiable conditions for reachability and controllability are also presented.
\end{abstract}

Key Words: Switched systems, discrete-time, controllability, reachability, reversible systems.

\section{Introduction}

Switched systems are hybrid systems that consist of two or more subsystems and are controlled by switching laws. The switching law may be either supervised or unsupervised, time-driven or event-driven. In this paper, we focus on the class of switched systems in which the switching laws are design parameters to be chosen online by a supervisor $[6,14,17]$.

Switched systems deserve investigation for theoretical interest as well as for practical applications. Switching among different system structures is an essential feature of many engineering control applications such as power systems and power electronics [15, 10]. Control techniques based on switching between different controllers have been investigated in recent years, particularly in the context of adaptive control [8, 9]. The existence of systems that cannot be asymptotically stabilized by a single continuous feedback controller also motivates us to study switched systems [2]. Switched systems also arise naturally in the study of multi-rate sample-data systems [11].

In the analysis and design of control systems, controllability and reachability are two fundamental concepts that need to be investigated. For switched continuous-time systems, the controllability and reachability issues have been addressed in several references. Studies for second-order switched linear systems can be found in $[7,17]$. Geometric tests for reachability were presented for general switched linear control systems in [13]. For switched linear discrete-time systems, the set of points reachable from the origin were investigated in [12]. It is shown that this set (termed controllable set in [12]) is a subspace under certain hypothesis, but not always the case in general. Some further extension

${ }^{*}$ To whom all correspondence should be addressed. Tel. (+65) 8746821; Fax. (+65) 7791103; E-mail: elegesz@nus.edu.sg 
of this work can be found in [3], where the controllable set as the union of its maximal components was investigated.

In this paper, the controllability and reachability issues are addressed for switched linear discrete-time systems. Following Wonham's geometric approach, multi-pair invariant subspaces were introduced. The relationships among the controllable set, the reachable set and the multi-pair invariant subspace are investigated. For reversible systems, verifiable criteria for controllability and reachability are presented.

The paper is organized as follows. Section 2 formulates the problem and presents preliminary analysis. Geometric characterizations for the controllability and reachability sets are presented in Section 3. In Section 4, two illustrating examples are given. Finally, some concluding remarks are made in the last section.

\section{Definitions and Preliminaries}

Consider a switched linear discrete-time control system given by

$$
x_{k+1}=A_{\sigma} x_{k}+B_{\sigma} u_{k}
$$

where $x_{k} \in \Re^{n}$ and $u_{k} \in \Re^{p}$ are the states and inputs, $\sigma:\{0,1, \cdots\} \rightarrow M=\{1,2, \cdots, m\}$ is the switching path to be designed, matrix pair $\left(A_{k}, B_{k}\right)$ for $1 \leq k \leq m$ are referred to as subsystems of (1).

For clarity, let us denote $\underline{\mathrm{k}}=\{0, \cdots, k-1\}$ for a positive integer $k$. It can be calculated that

$$
x_{k}=\left(\Pi_{j=0}^{k-1} A_{i_{j}}\right) x_{0}+\left(\Pi_{j=1}^{k-1} A_{i_{j}}\right) B_{i_{0}} u_{0}+\cdots+A_{i_{k-1}} B_{i_{k-2}} u_{k-2}+B_{i_{k-1}} u_{k-1}
$$

where $i_{j}=\sigma(j)$ for $j=0, \cdots, k-1$.

A state configuration $x$ is said controllable, if it is transferable to the origin in finite time by appropriate choices of input $u$ and switching path $\sigma$. The precise definitions of the relevant concepts are given as follows.

Definition 1 State $x \in \Re^{n}$ is controllable, if there exist a time instant $k>0$, a switching path $\sigma:\{0, \cdots, k-1\} \rightarrow M$, and inputs $u: \underline{k} \rightarrow \Re^{p}$, such that $x_{0}=x$ and $x_{k}=0$.

Definition 2 The controllable set of system (1) is the set of states which are controllable.

Definition 3 System (1) is said (completely) controllable, if its controllable set is $\Re^{n}$.

For any matrices $A \in \Re^{n \times n}$ and $B \in \Re^{n \times p}$, denote $\mathcal{B}=\operatorname{Im} B$ and $A^{-1} \mathcal{B}=\left\{x \in \Re^{n}: A x \in\right.$ $\mathcal{B}\}$. Define

$$
\mathcal{C}\left(i_{0}, \cdots, i_{k}\right)=\left(A_{i_{k}} \cdots A_{i_{0}}\right)^{-1}\left(A_{i_{k}} \cdots A_{i_{1}} \mathcal{B}_{i_{0}}+\cdots+A_{i_{k}} \mathcal{B}_{i_{k-1}}+\mathcal{B}_{i_{k}}\right)
$$


Let $\mathcal{C}_{k}$ denote the set of points which can be transfered to the origin within $k$ steps. It can be readily seen that

$$
\mathcal{C}_{k}=\cup_{i_{0}, \cdots, i_{k-1} \in M} \mathcal{C}\left(i_{0}, \cdots, i_{k-1}\right)
$$

and

$$
\mathcal{C}=\cup_{k=1}^{\infty} \mathcal{C}_{k}
$$

where $\mathcal{C}$ is the controllable set of system (1).

The reachability counterparts can be defined along the same line as follows.

Definition 4 State $x \in \Re^{n}$ is reachable, if there exist a time instant $k>0$, a switching path $\sigma: \underline{k} \rightarrow M$, and inputs $u: \underline{k} \rightarrow \Re^{p}$, such that $x_{0}=0$ and $x_{k}=x$.

Definition 5 The reachable set of system (1) is the set of states which are reachable.

Definition 6 System (1) is said (completely) reachable, if its reachable set is $\Re^{n}$.

Define

$$
\mathcal{R}\left(i_{0}, \cdots, i_{k}\right)=A_{i_{k}} \cdots A_{i_{1}} \mathcal{B}_{i_{0}}+\cdots+A_{i_{k}} \mathcal{B}_{i_{k-1}}+\mathcal{B}_{i_{k}}
$$

Let $\mathcal{R}_{k}$ denote the set of points which are reachable from the origin within $k$ steps. It can be readily seen that

$$
\mathcal{R}_{k}=\cup_{i_{0}, \cdots, i_{k-1} \in M} \mathcal{R}\left(i_{0}, \cdots, i_{k-1}\right)
$$

and

$$
\mathcal{R}=\cup_{k=1}^{\infty} \mathcal{R}_{k}
$$

where $\mathcal{R}$ is the reachable set of system (1).

Given matrix $A$ and subspace $\mathcal{B} \in \Re^{n}$, let $\Gamma_{A} \mathcal{B}$ denote the minimal $A$-invariant subspace that contains $\mathcal{B}$, i.e.,

$$
\Gamma_{A} \mathcal{B}=\mathcal{B}+A \mathcal{B}+\cdots+A^{n-1} \mathcal{B}
$$

This operation can be defined recursively as $\Gamma_{A_{1}} \Gamma_{A_{2}} \mathcal{B}=\Gamma_{A_{1}}\left(\Gamma_{A_{2}} \mathcal{B}\right)$. For clarity, define the nested subspaces as

$$
\begin{aligned}
\mathcal{V}_{1} & =\operatorname{Im} B_{1}+\cdots+\operatorname{Im} B_{m} \\
\mathcal{V}_{i+1} & =\Gamma_{A_{1}} \mathcal{V}_{i}+\cdots+\Gamma_{A_{m}} \mathcal{V}_{i} \quad i=1,2, \cdots
\end{aligned}
$$

and

$$
\mathcal{V}=\sum_{k=1}^{\infty} \mathcal{V}_{k}
$$


Note that if $\operatorname{dim} \mathcal{V}_{j}=\operatorname{dim} \mathcal{V}_{j+1}$, then $\mathcal{V}_{l}=\mathcal{V}_{j}$ for $l>j$. This fact implies that $\mathcal{V}_{n}=\mathcal{V}$. It is readily seen that this subspace is the minimum subspace which is invariant under $A_{i}$, $i=1, \cdots, m$ and contains $\sum_{j=1}^{m} \mathcal{B}_{j}$. Subspace $\mathcal{V}$ plays an important role in the following derivations.

Because $A^{i} \operatorname{Im} B \subseteq \Gamma_{A} \operatorname{Im} B$ for all $A \in \Re^{n \times n}, B \in \Re^{n \times p}$ and $i \geq 0$, we know that the reachable set

$$
\mathcal{R} \subseteq \cup_{k=0}^{\infty} \cup_{i_{0}, \cdots, i_{k-1} \in M}\left(\Gamma_{A_{i_{k-1}}} \cdots \Gamma_{A_{i_{1}}} \mathcal{B}_{i_{0}}+\cdots+\mathcal{B}_{i_{k-1}}\right) \subseteq \mathcal{V}
$$

System (1) is said to be reversible, if all matrices $A_{i}, i=1, \cdots, m$ are nonsingular. For a reversible system, the controllable set

$$
\mathcal{C} \subseteq \cup_{k=0}^{\infty} \cup_{i_{0}, \cdots, i_{k-1} \in M}\left(\Gamma_{A_{i_{0}}^{-1}} \mathcal{B}_{i_{0}}+\cdots+\Gamma_{A_{i_{0}}^{-1}} \cdots \Gamma_{A_{i_{k-1}}^{-1}} \mathcal{B}_{i_{k-1}}\right) \subseteq \mathcal{V}
$$

The above analysis are summarized in the following proposition.

Proposition 1 If switched linear system (1) is reachable, or if system (1) is reversible and controllable, then

$$
\mathcal{V}=\Re^{n}
$$

\section{Main Results}

\subsection{Geometric Characterizations}

In this subsection, we shall present a criterion of controllability for switched linear systems.

Theorem 1 Switched linear system (1) is controllable if, and only if there exist an integer $k<\infty$, and $i_{0}, \cdots, i_{k}$, such that

$$
\operatorname{Im}\left(A_{i_{k}} \cdots A_{i_{1}} A_{i_{0}}\right) \subseteq \mathcal{R}\left(i_{0}, \cdots, i_{k}\right)
$$

Proof. From (3),(4) and (5),the controllable set of system (1) is given by

$$
\mathcal{C}=\cup_{k=1}^{\infty} \cup_{i_{0}, \cdots, i_{k-1} \in M}\left(\left(A_{i_{k-1}} \cdots A_{i_{0}}\right)^{-1}\left(A_{i_{k-1}} \cdots A_{i_{1}} \mathcal{B}_{i_{0}}+\cdots+A_{i_{k-1}} \mathcal{B}_{i_{k-2}}+\mathcal{B}_{i_{k-1}}\right)\right)
$$

That is, the controllable set can be expressed as countable unions of subspaces of $\Re^{n}$. Because $\Re^{n}$ cannot be expressed as countable unions of lower-dimensional subspaces, to ensure controllability of system (1), it must have

$$
\mathcal{C}\left(i_{0}, \cdots, i_{k}\right)=\left(A_{i_{k}} \cdots A_{i_{0}}\right)^{-1}\left(A_{i_{k}} \cdots A_{i_{1}} \mathcal{B}_{i_{0}}+\cdots+A_{i_{k}} \mathcal{B}_{i_{k-1}}+\mathcal{B}_{i_{k}}\right)=\Re^{n}
$$


for some $k<\infty$ and $i_{0}, \cdots, i_{k} \in M$. That is

$$
\left(A_{i_{k}} \cdots A_{i_{1}} \mathcal{B}_{i_{0}}+\cdots+A_{i_{k}} \mathcal{B}_{i_{k-1}}+\mathcal{B}_{i_{k}}\right) \supseteq \operatorname{Im}\left(A_{i_{k}} \cdots A_{i_{0}}\right) \quad \diamond
$$

For reachability of switched linear systems, similar criterion can be found in [12, Corollary of Theorem 2]. It is summarized in the following theorem for completeness.

Theorem 2 Switched linear system (1) is reachable if, and only if there exist an integer $k<\infty$, and $i_{0}, \cdots, i_{k}$, such that

$$
\mathcal{R}\left(i_{0}, \cdots, i_{k}\right)=\Re^{n}
$$

Remark 1 It is interesting to notice the resemblance between reachability of a switched linear system and weak controllability of a jump linear system [5]. For a jump linear system [5]

$$
x_{k+1}=A_{r_{k}} x_{k}+B_{r_{k}} u_{k}
$$

where $r_{k} \in M=\{1, \cdots, m\}, k=1,2, \cdots$ forms a finite-state discrete-time ergodic Markov chain, it is weakly controllable if and only if for some $r_{i_{0}} \in M$, there exists a possible transition sequence $i_{0}, \cdots, i_{T-1}$ with $T \leq \infty$, such that

$$
\operatorname{rank}\left[B_{i_{T-1}}, A_{i_{T-1}} B_{i_{T-2}}, \cdots, A_{i_{T-1}} \cdots A_{i_{1}} B_{i_{0}}\right]=n
$$

which is equivalent to condition (13).

As proved in [4], any causal discrete-time (input-output) system can be realized with a reversible state variable representation. Accordingly, reversible system representation is very general and applicable to a large class of systems. In the sequel, we present verifiable criteria for controllability and reachability of reversible switched linear systems. Moreover, we prove that the reachable and controllable sets are nothing but subspace $\mathcal{V}$ in this case.

Theorem 3 Suppose switched linear system (1) is reversible, then its reachable set is

$$
\mathcal{R}=\mathcal{V}
$$

Proof. Let us prove it by contradiction. Suppose

$$
\operatorname{dim} \mathcal{R}\left(i_{0}, \cdots, i_{k}\right)=\max \left\{\operatorname{dim} \mathcal{R}\left(l_{0}, \cdots, l_{j}\right): l_{0}, \cdots, l_{j} \in M, j=0,1, \cdots\right\}<\operatorname{dim} \mathcal{V}
$$

For any arbitrary given integers $l_{0}, \cdots, l_{j}$, consider the subspace

$$
\begin{aligned}
\mathcal{R}\left(l_{0}, \cdots, l_{j}, i_{0}, \cdots, i_{k}\right)= & A_{i_{k}} \cdots A_{i_{0}} A_{l_{j}} \cdots A_{l_{1}} \mathcal{B}_{l_{0}}+\cdots+A_{i_{k}} \cdots A_{i_{0}} \mathcal{B}_{l_{j}} \\
& +A_{i_{k}} \cdots A_{i_{1}} \mathcal{B}_{i_{0}}+\cdots+\mathcal{B}_{i_{k}}
\end{aligned}
$$


It follows from (16) that

$$
\operatorname{dim} \mathcal{R}\left(l_{0}, \cdots, l_{j}, i_{0}, \cdots, i_{k}\right)=\operatorname{dim} \mathcal{R}\left(i_{0}, \cdots, i_{k}\right)
$$

which implies that

$$
\left(A_{i_{k}} \cdots A_{i_{0}}\right)\left(A_{l_{j}} \cdots A_{l_{1}} \mathcal{B}_{l_{0}}+\cdots+\mathcal{B}_{l_{j}}\right) \subseteq \mathcal{R}\left(i_{0}, \cdots, i_{k}\right)
$$

which further implies that

$$
\left(A_{i_{k}} \cdots A_{i_{0}}\right)\left(A_{l_{j}} \cdots A_{l_{1}} \mathcal{B}_{l_{0}}\right) \subseteq \mathcal{R}\left(i_{0}, \cdots, i_{k}\right)
$$

On the other hand, note that

$$
\mathcal{V}=\mathcal{V}_{n}=\sum_{i_{1}, \cdots, i_{n}=1, \cdots, m}^{j_{1}, \cdots, j_{n}=0,1, \cdots, n-1} A_{i_{n}}^{j_{n}} \cdots A_{i_{1}}^{j_{1}} \mathcal{B}_{i_{1}}
$$

Since $j$ and $l_{0}, \cdots, l_{j}$ in (17) can take arbitrarily any values, we have

$$
\left(A_{i_{k}} \cdots A_{i_{0}}\right) \mathcal{V} \subseteq \mathcal{R}\left(i_{0}, \cdots, i_{k}\right)
$$

which is a contradiction because

$$
\operatorname{dim}\left[\left(A_{i_{k}} \cdots A_{i_{0}}\right) \mathcal{V}\right]=\operatorname{dim} \mathcal{V}>\operatorname{dim} \mathcal{R}\left(i_{0}, \cdots, i_{k}\right)
$$

where the equality follows from the identity $\operatorname{dim} A \mathcal{V}=\operatorname{dim} \mathcal{V}$ for any nonsingular matrix $A \in \Re^{n \times n}$ and subspace $\mathcal{V} \subseteq \Re^{n}$.

Accordingly, we have

$$
\operatorname{dim} \mathcal{R}\left(i_{0}, \cdots, i_{k}\right)=\operatorname{dim} \mathcal{V}
$$

Since

$$
\mathcal{R}\left(i_{0}, \cdots, i_{k}\right) \subseteq \mathcal{R} \subseteq \mathcal{V}
$$

we thus have

$$
\mathcal{R}=\mathcal{V}
$$

Theorem 4 Suppose switched linear system (1) is reversible, then its controllable set is

$$
\mathcal{C}=\mathcal{V}
$$

Proof. This theorem can be proven following the same argument of the proof of Theorem 3 and the details are omitted. 
Corollary 1 For a reversible switched linear system, the following statements are equivalent:

(i) The system is completely controllable;

(ii) The system is completely reachable; and

(iii) $\mathcal{V}=\Re^{n}$.

Proof. Follows directly from Theorems 3 and 4.

Remark 2 For reversible switched linear systems, the reachable set, the controllable set and subspace $\mathcal{V}$ always coincide with each other. For non-reversible systems, however, these favorable properties do not hold any more as shown in Example 1 of Section 4.

Remark 3 The criterion for controllability and reachability in Corollary 1 is an extension of the well known geometric criterion for reachability of linear system $(A, B)[16]$

$$
\operatorname{Im} B+A \operatorname{Im} B+\cdots+A^{n-1} \operatorname{Im} B=\Re^{n}
$$

It is equivalent to the Kalman-type rank condition

$$
\begin{gathered}
\operatorname{rank}\left[B_{1}, \cdots, B_{m}, A_{1} B_{1}, \cdots, A_{1} B_{m}, \cdots, A_{m} B_{1}, \cdots, A_{m} B_{m}, \cdots, A_{1}^{n-1} B_{1}, \cdots,\right. \\
\left.A_{1}^{n-1} B_{m}, A_{1}^{n-2} A_{2} B_{1}, \cdots, A_{1}^{n-2} A_{2} B_{m}, \cdots, A_{m}^{n-1} B_{1}, \cdots, A_{m}^{n-1} B_{m}\right]=n
\end{gathered}
$$

which can be efficiently verified by polynomial-time algorithms [1].

\subsection{Computational Issues}

As stated in Theorems 3 and 4, the controllable (reachable) set for a reversible switched system is $\mathcal{V}$, which is defined recursively through $\left(A_{i}, B_{i}\right), i=1, \cdots, m$. The quantity relationship between them is

$$
\mathcal{V}=\sum_{i_{1}, \cdots, i_{n}=1, \cdots, m}^{j_{1}, \cdots, j_{n}=0,1, \cdots, n-1} A_{i_{n}}^{j_{n}} \cdots A_{i_{1}}^{j_{1}} \mathcal{B}_{i_{1}}
$$

That is, $\mathcal{V}$ is summation of $(m n)^{n} p$ items. It requires large computational efforts to calculate this subspace if $m$ and $n$ are relatively large.

In this subsection, we provide a procedure to calculate $\mathcal{V}$ more efficiently.

Denote the nested subspaces as

$$
\begin{aligned}
& \mathcal{W}_{0}=\mathcal{B}_{1}+\cdots+\mathcal{B}_{m} \\
& \mathcal{W}_{i}=\mathcal{W}_{i-1}+A_{1} \mathcal{W}_{i-1}+\cdots+A_{m} \mathcal{W}_{i-1}, \quad i=1,2, \cdots
\end{aligned}
$$


and furthermore

$$
\mathcal{W}=\sum_{i=0}^{\infty} \mathcal{W}_{i}
$$

It can be readily seen that $\mathcal{V}_{n} \subseteq \mathcal{W} \subseteq \mathcal{V}$. As a consequence, $\mathcal{W}=\mathcal{V}$. Let $\rho$ denote the minimal integer $j$ such that $\mathcal{W}_{j}=\mathcal{W}$. It can be readily seen that $\rho \leq n-1$. Define natural numbers

$$
n_{0}=\operatorname{dim} \mathcal{W}_{0}, \quad n_{i}=\operatorname{dim} \mathcal{W}_{i}-\operatorname{dim} \mathcal{W}_{i-1}, i=1, \cdots, \rho
$$

and furthermore

$$
\tau_{i}=\sum_{j=0}^{i} n_{j}, i=1, \cdots, \rho
$$

A basis of subspace $\mathcal{V}$ can be constructed according to the following steps:

1. Choose a group of base vectors $\gamma_{1}, \cdots, \gamma_{n_{0}}$ in $\mathcal{W}_{0}$.

2. Because

$$
\mathcal{W}_{1}=\mathcal{W}_{0}+\sum_{i=1}^{m} A_{i} \mathcal{W}_{0}=\operatorname{span}\left\{\gamma_{j}, A_{i} \gamma_{j}, i=1, \cdots, m, j=1, \cdots, n_{0}\right\}
$$

we can find a basis of $\mathcal{W}_{1}$ by searching the set

$$
\left\{\gamma_{1}, \cdots, \gamma_{n_{0}}, A_{1} \gamma_{1}, \cdots, A_{1} \gamma_{n_{0}}, \cdots, A_{m} \gamma_{1}, \cdots, A_{m} \gamma_{n_{0}}\right\}
$$

from left to right for linearly independent column vectors. Denote this basis as

$$
\gamma_{1}, \cdots, \gamma_{n_{0}}, \gamma_{n_{0}+1}, \cdots, \gamma_{\tau_{1}}
$$

3. Continuing the process, we can find a basis $\gamma_{1}, \cdots, \gamma_{n_{0}}, \cdots \gamma_{\tau_{l-1}+1}, \cdots, \gamma_{\tau_{l}}$ for $\mathcal{W}_{l}$. Because

$$
\mathcal{W}_{l+1}=\mathcal{W}_{l}+\sum_{i=1}^{m} A_{i} \mathcal{W}_{l}=\operatorname{span}\left\{\gamma_{1}, \cdots, \gamma_{\tau}, A_{i} \gamma_{j}, i=1, \cdots, m, j=\tau_{l-1}+1, \cdots, \tau_{l}\right\}
$$

by searching the set

$$
\left\{\gamma_{1}, \cdots, \cdots, \gamma_{\tau_{l}}, A_{1} \gamma_{\tau_{l-1}+1}, \cdots, A_{1} \gamma_{\tau_{l}}, \cdots, A_{m} \gamma_{\tau_{l-1}+1}, \cdots, A_{m} \gamma_{\tau_{l}}\right\}
$$

from left to right for linearly independent column vectors, we can find a basis of $\mathcal{W}_{l+1}$ :

$$
\gamma_{1}, \cdots, \gamma_{\tau_{l}}, \gamma_{\tau_{l}+1}, \cdots, \gamma_{\tau_{l+1}}
$$

4. Finally, we can find a basis $\gamma_{1}, \cdots, \gamma_{\tau_{\rho}}$ of subspace $\mathcal{W}$. That is,

$$
\mathcal{V}=\mathcal{W}=\operatorname{span}\left\{\gamma_{1}, \cdots, \gamma_{\tau_{\rho}}\right\}
$$

It involves not more than $m p+m \tau_{\rho-1}$ column vectors in the above procedure, which is only a small fraction of the original quantity, $(m n)^{n} p$. 


\subsection{Observability and Reconstructibility}

In the above analysis, reference is made to reachability and controllability only. It should be noticed that the observability and reconstructibility counterparts can be addressed dualistically. In the sequel, we outline the relevant concepts and the corresponding criteria.

Consider a switched linear discrete-time control system with outputs given by

$$
\begin{aligned}
& x_{k+1}=A_{\sigma} x_{k}+B_{\sigma} u_{k} \\
& y_{k}=E_{\sigma} x_{k}
\end{aligned}
$$

where $x_{k} \in \Re^{n}, u_{k} \in \Re^{p}$ and $y_{k} \in \Re^{r}$ are the states, inputs and outputs, respectively, and $\sigma:\{0,1, \cdots\} \rightarrow M=\{1,2, \cdots, m\}$ is the switching path to be designed.

Definition 7 The switched linear system (23) is (completely) observable, if there exist an integer $k$ and a switching path $\sigma: \underline{k} \rightarrow M$, such that knowledge of the output sequence $\left\{y_{0}, y_{1}, \cdots, y_{k}\right\}$ and the input sequence $\left\{u_{0}, u_{1}, \cdots, u_{k-1}\right\}$ is sufficient to determine $x_{0}$.

Definition 8 The switched linear system (23) is (completely) reconstructible, if there exist an integer $k$ and a switching path $\sigma: \underline{k} \rightarrow M$, such that state $x_{k}$ can be determined from knowledge of the output sequence $\left\{y_{0}, \cdots, y_{k}\right\}$ and the input sequence $\left\{u_{0}, \cdots, u_{k-1}\right\}$.

In view of Theorems 1-4 for reachability and controllability, the following criteria are readily obtained for observability and reconstructibility by using the principle of duality.

Theorem 5 Switched linear system (23) is observable if, and only if there exist an integer $k<\infty$, and $i_{0}, \cdots, i_{k}$, such that

$$
\mathcal{E}_{i_{0}}+A_{i_{0}}^{T} \mathcal{E}_{i_{1}}+\cdots+A_{i_{0}}^{T} \cdots A_{i_{k-1}}^{T} \mathcal{E}_{i_{k}}=\Re^{n}
$$

where $\mathcal{E}_{i}=\operatorname{Im}_{i}^{T}$ for $i=1, \cdots, m$.

Moreover, if the system is reversible, then a necessary and sufficient condition for observability is

$$
\sum_{i_{1}, \cdots, i_{n}=1, \cdots, m}^{j_{1}, \cdots, j_{n}=0,1, \cdots, n-1}\left(A_{i_{1}}^{j_{1}}\right)^{T} \cdots\left(A_{i_{n-1}}^{j_{n-1}}\right)^{T} \mathcal{E}_{i_{n}}=\Re^{n}
$$

Theorem 6 Switched linear system (23) is reconstructible if, and only if there exist an integer $k<\infty$, and $i_{0}, \cdots, i_{k}$, such that

$$
\mathcal{E}_{i_{0}}+A_{i_{0}}^{T} \mathcal{E}_{i_{1}}+\cdots+A_{i_{0}}^{T} \cdots A_{i_{k-1}}^{T} \mathcal{E}_{i_{k}} \supseteq \operatorname{Im}\left(A_{i_{0}}^{T} \cdots A_{i_{k}}^{T}\right)
$$

Moreover, if the system is reversible, then (25) is a necessary and sufficient condition for reconstructibility. 


\section{Illustrating Examples}

Example 1 Consider system (1) with $n=4, m=2$, and

$$
A_{1}=\left[\begin{array}{llll}
0 & 0 & 0 & 0 \\
0 & 0 & 0 & 0 \\
0 & 0 & 1 & 0 \\
0 & 0 & 1 & 0
\end{array}\right], B_{1}=\left[\begin{array}{l}
1 \\
0 \\
0 \\
0
\end{array}\right] ; A_{2}=\left[\begin{array}{llll}
0 & 0 & 0 & 0 \\
1 & 0 & 0 & 0 \\
0 & 0 & 0 & 1 \\
0 & 0 & 0 & 1
\end{array}\right], B_{2}=\left[\begin{array}{l}
0 \\
0 \\
0 \\
0
\end{array}\right]
$$

Simple calculation gives

$$
\begin{aligned}
\mathcal{V} & =\operatorname{span}\left\{e_{1}, e_{2}\right\} \\
\mathcal{R} & =\operatorname{span}\left\{e_{1}\right\} \cup \operatorname{span}\left\{e_{2}\right\} \\
\mathcal{C} & =\operatorname{span}\left\{e_{1}, e_{2}, e_{3}\right\} \cup \operatorname{span}\left\{e_{1}, e_{2}, e_{4}\right\}
\end{aligned}
$$

Note that neither the controllable set nor the reachable set is a subspace of the total space. Furthermore, $\mathcal{R} \subset \mathcal{V} \subset \mathcal{C}$, where the subset relationships are strict proper.

Example 2 Controllability of a multi-rate sampled-data system.

Consider the linear continuous time-invariant system given by

$$
\dot{x}=A x+B u(t)=\left[\begin{array}{cccc}
0 & -100 \pi & 0 & 0 \\
100 \pi & 0 & 0 & 0 \\
0 & 0 & \frac{3}{2} \pi & 0 \\
0 & 0 & 0 & \frac{3}{2} \pi
\end{array}\right]+\left[\begin{array}{cc}
10 & 0 \\
10 & 0 \\
0 & 10 \\
0 & 10
\end{array}\right] u(t)
$$

which can be verified to be controllable.

The corresponding sampled-data system is given by

$$
x_{k+1}=A_{T} x_{k}+B_{T} u_{k}
$$

where $T$ is the sampling interval,

$$
x_{k}=x(k T), \quad u_{k}=u(k T), \quad A_{T}=e^{A T}, \quad B_{T}=\int_{0}^{T} e^{\tau A} d \tau B
$$

If the sampling intervals are choosing as $T_{1}=0.01$ and $T_{2}=0.015$, we have the corresponding matrix pairs $\left(A_{T_{1}}, B_{T_{1}}\right)$ and $\left(A_{T_{2}}, B_{T_{2}}\right)$ given as

$$
A_{T_{1}}=\left[\begin{array}{cccc}
-1 & 0 & 0 & 0 \\
0 & -1 & 0 & 0 \\
0 & 0 & -0.5 & -\sin \frac{3}{2} \pi \\
0 & 0 & \sin \frac{3}{2} \pi & -0.5
\end{array}\right], \quad B_{T_{1}}=\left[\begin{array}{cc}
0.1 & 0 \\
0.1 & 0 \\
0 & -0.05-0.1 \sin \frac{3}{2} \pi \\
0 & -0.05-0.1 \sin \frac{3}{2} \pi
\end{array}\right]
$$


and

$$
A_{T_{2}}=\left[\begin{array}{cccc}
0 & 1 & 0 & 0 \\
-1 & 0 & 0 & 0 \\
0 & 0 & -1 & 0 \\
0 & 0 & 0 & -1
\end{array}\right], \quad B_{T_{2}}=\left[\begin{array}{cc}
0.15 & 0 \\
-0.15 & 0 \\
0 & -0.15 \\
0 & -0.15
\end{array}\right]
$$

It can be verified that

$$
\operatorname{rank}\left[B_{T_{i}}, A_{T_{i}} B_{T_{i}}, \cdots, A_{T_{i}}^{3} B_{T_{i}}\right]=3, \quad i=1,2
$$

which shows that the corresponding sampled-data systems are not controllable.

Now we consider the multi-rate sampling of system (28) with sampling rate of either $T_{1}$ or $T_{2}$. A question naturally arise: Does there exist a sampling strategy such that the resulted switched system is controllable? That is, whether switched system (1) with $A_{i}=A_{T_{i}}, \quad B_{i}=B_{T_{i}}, \quad i=1,2$ is controllable or not?

Simple computation gives

$$
\mathcal{V} \supseteq \operatorname{span}\left\{B_{1}, B_{2}, A_{1} B_{1}, A_{2} B_{2}\right\}=\Re^{4}
$$

From Corollary 1, the controllability follows. Moreover, it can be verified that

$$
\mathcal{C}(2,1)=\mathcal{R}(2,1)=\Re^{4}
$$

Accordingly, the switched system is controllable from (and reachable to) any point within 2 steps by choosing subsystem $\left(A_{2}, B_{2}\right)$ at the first step and then switching to subsystem $\left(A_{1}, B_{1}\right)$ at the second step.

This example illustrates that switching among different sampling rates may avoid singularities caused by inappropriate choice of sampling rates.

\section{Conclusion}

Controllability and reachability issues have been addressed for switched linear discretetime systems. Geometric characterizations for controllability and reachability were presented. For reversible systems, the controllable and reachable sets are proven to be subspaces of the total space, and verifiable criteria for controllability and reachability have also been presented. Criteria for observability and reconstructibility have also been presented.

\section{ACKNOWLEDGEMENT}

The authors would like to thank the reviewers for their constructive and insightful comments for further improving the quality of this work. We appreciate D. P. Stanford' kind help in providing one of his papers. 


\section{References}

[1] V. D. Blondel and J. N. Tsitsiklis, "A survey of computational complexity results in systems and control," Automatica, vol. 36, pp. 1249-1274, 2000.

[2] R. W. Brockett, "Asymptotic stability and feedback stabilization," in Differential Geometric Control Theory, R. W. Brockett et al, eds, Boston: Birkhauser, 1983, pp. $181-191$.

[3] L. T. Conner,Jr. and D. P. Stanford, "The structure of controllable set for multimodal systems," Linear Algebra Applic., vol. 95, pp.171-180, 1987.

[4] M. Fliess, "Reversible linear and nonlinear discrete-time dynamics," IEEE Trans. Automat. Contr., vol. 37, pp. 1144-1153, 1992.

[5] Y. Ji and H. J. Chizeck, "Controllability, observability and discrete-time markovian jump linear quadratic control," Int. J. Contr., vol. 48, pp. 481-498, 1988.

[6] D. Liberzon and A. S. Morse, "Basic problems in stability and design of switched systems," IEEE Contr. Syst. Mag., vol. 19, no. 5, pp. 59-70, 1999.

[7] K. A. Loparo, J. T. Aslanis and O. IIajek, "Analysis of switching linear systems in the plain, part 2, global behavior of trajectories, controllability and attainability," $J$. of Optim. Theory Appl., vol. 52, pp. 395-427, 1987.

[8] A. S. Morse, "Supervisory control of families of linear set-point controllers- Part 1:, exact matching," IEEE Trans. Automat. Contr., vol.41, pp. 1413-1431, 1996.

[9] K. S. Narendra and J. Balakrishnan, "Adaptive control using multiple models," IEEE Trans. Automat. Contr., vol.42, pp. 171-187, 1997.

[10] H. Sira-Ramirez, "Nonlinear P-I controller design for switch mode dc-to-dc power converters," IEEE Trans. Circuits Syst., vol. 38, pp. 410-417, 1991.

[11] D. P. Stanford, "Stability for a multi-rate sampled-datd system," SIAM J. Contr. Optimiz., vol.17, pp.390-399, 1979.

[12] D. P. Stanford and L. T. Conner, Jr., "Controllability and stabilizability in multi-pair systems," SIAM J. Contr. Optimiz., vol. 18, pp. 488-497, 1980.

[13] Z. Sun and D. Z. Zheng, "On reachability and stabilization of switched linear control systems," to appear in IEEE Trans. Automat. Contr., 2001.

[14] M. A. Wicks, P. Peleties and R. A. DeCarlo, "Switched controller synthesis for the quadratic stabilization of a pair of unstable linear systems," Euro. J. Contr., vol. 4, pp. 140-147, 1998.

[15] S. M. Williams and R. G. Hoft, "Adaptive frequency domain control of PPM switched power line conditioner," IEEE Trans. Power Electron, vol. 6, pp. 665-670, 1991.

[16] W. M. Wonham, Linear Multivariable Control - A Geometric Approach. Berlin: Spinger-Verlag, 1974.

[17] X. Xu and P. J. Antsaklis, "On the reachability of a class of second-order switched systems," Technical report, ISIS-99-003, University of Notre Dame, February, 1999. 